\title{
Columnar versus smectic order in systems of charged colloidal rods
}

\author{
H. H. Wensink* \\ Institut für Theoretische Physik, Heinrich-Heine-Universität Düsseldorf, \\ Universitätsstraße 1, D-40225, Düsseldorf, Germany
}

(Dated: April 2, 2018)

\begin{abstract}
We study the stability of inhomogeneous liquid crystalline states in systems of monodisperse, stiff, charged rods. By means of a bifurcation analysis applied to the Onsager free energy for charged rods in strongly nematic states, we investigate nematic-smectic and nematic-columnar instabilities as a function of the Debye screening length $\kappa^{-1}$. While the nematic-smectic transition clearly pre-emts the nematic-columnar one in the regime of strong screening (i.e. small $\kappa^{-1}$ ) a marked stability of hexagonal columnar order is observed at larger screening lengths. The theoretical results are substantiated by Brownian dynamics computer simulation results based on the Yukawa-site model. Our findings connect to experiments on tobacco mosaic virus rods in particular but might be relevant for soft rod-like mesogens in strong external directional fields in general.
\end{abstract}

PACS numbers: 64.70.Md,82.70.Dd,61.20.Ja

\section{INTRODUCTION}

Over the past decades, much research effort has been devoted to understanding the liquid crystal phase behavior of hard non-spherical colloidal particles, particularly in density functional theory and computer simulations. The theoretical approach to lyotropic liquid crystal formation has been initiated a long time ago by Onsager $\underline{1}$ with his classic paper on the isotropic-nematic transition of infinitely thin rods. This theory shows that repulsive interactions alone can lead to long-range orientational (nematic) order, disproving the notion that attractive interactions are a prerequisite. The full phase diagram for hard spherocylinders with variable aspect ratio has only fairly recently been mapped out completely by means of extensive computer simulations ${ }^{2,3}$. A notable feature is that nematic order is only stable for sufficiently large aspect ratios while isotropic systems of short rods tend to freeze directly into a crystalline state with three-dimensional long-range positional order. As to the nematic state, additional high-density phase transitions may occur involving partial freezing into liquid crystals with long-range spatial inhomogeneities in either one dimension, giving a smectic A $(\mathrm{SmA})$ phase, or two dimensions leading to columnar $(\mathrm{C})$ order. The latter also plays a dominant role in the high-density phase behavior of plate-like colloids where a stable hexagonal columnar was found in simulations 4,5 and experimental studies of model clay suspensions 6.7 .

Complicating issues, often important in interpreting experimental results, include the effect of rod flexibility ${ }^{8.9}$

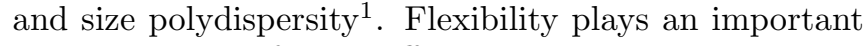
role in systems of e.g. stiff polymers and linear micelles and its generic effect is a significant depression of nematic order compared to rigid particles $\frac{10}{}$. Systems of self-assembling worm-like micelles may, under certain circumstances, not show nematic order at all. Instead a phase transition from an isotropic fluid to a hexagonal columnar phase takes place $\frac{11.12}{}$. The stability of the columnar state is also strongly stimulated by the inher- ent length polydispersity which may be of the annealed form, where the size distribution depends upon density such as in micellar systems, or of the quenched type like for many colloidal systems where the size distribution is fixed by the synthesis procedure. A simple packing argument suffices to understand that rods with variable length do not easily fit into layers pertaining to smectic order and therefore prefer columnar order. The crossover to columnar order has been observed explicitly in binary mixtures of hard rods with two different lengths $\frac{13,14}{}$.

Another important factor in the phase stability of rodlike mesogens is the influence of the soft repulsive interactions. In the experimental situation, interactions between colloidal model rods with chemically modified surfaces can never be rendered truly hard 15,16 and many important biomacromolecular systems such as tobacco mosaic virus (TMV) and $f d$ virus rods $\frac{17}{}$, or mineral systems $\frac{18}{18}$ like goethite $\frac{19}{}$ and vanadium pentoxide $\left(\mathrm{V}_{2} \mathrm{O}_{5}\right)^{20}$ rods are stabilized by electrostatic particle repulsions. The influence of these interactions on the isotropic-nematic transition had already been addressed by Onsager in his original paper. Later on, the effect of electrostatic 'twist', which disfavors parallel rod configurations and hence destabilizes nematic order, has been worked out in more detail in a study by Stroobants et $a l .21$.

Much more challenging however is the question how the electrostatic interactions affect the stability of the inhomogeneous liquid crystal phases, in particular the smectic phase, formed at high densities. Theoretical attempts in this direction have been undertaken in Ref. 22 and 23. Based on a model which combines the concept of an effective rod thickness (to account for the extent of the electric double layer) with a simple cell description to treat the inhomogeneous states, Kramer and Herzfeld ${ }^{22,24}$ were able to construct the phase diagram of parallel, charged rods. Their main conclusion, a generic charge-induced stabilization of the inhomogeneous liquid crystal states also shows up in the study of Graf and Löwen ${ }^{23}$ based on an elaborate density functional the- 
ory.

In this paper we present a similar study starting from the Onsager theory for freely rotating rods. Rather than attempting to construct the full phase diagram we will merely focus on locating symmetry-breaking instabilities of the nematic state towards the inhomogeneous phases. The main candidates are the smectic and the hexagonal columnar phases. The study of these type of instabilities has been pioneered a few decades ago by Mulder ${ }^{25,26}$ and the preferential stability of smectic over columnar order in systems of freely rotating hard rods, first recognized in simulations ${ }^{27}$, could also be established in theory ${ }^{28}$.

Here, we will elaborate on the Onsager-type approaches and extend the calculations towards charged systems in two steps. First, systems of parallel charged rods will be considered by employing a straightforward site model to account for the electrostatic end cap effects. Next, the restriction of parallel confinement will be removed and the influence of rotations and electrostatic twist shall be explicitly taken into account by considering the pair potential for infinitely stretched line charges. Although the second virial theory is not quantitatively valid for our case (the weight of parallel rod configurations which drive the translational symmetry-breaking instabilities necessitates inclusion of higher virial coefficients into the free energy), we expect the theory to give a reliable qualitative sketch of the competitive stability of smectic and columnar order for soft rods.

In contrast to the previous theoretical studies of Refs. 22 and 23, our calculations reveal a distinct crossover from smectic to columnar order upon decreasing ionic strength. These results may be helpful in interpreting experimental results on TMV rods at low ionic strength. Most importantly, we show that columnar stability in rod systems need not be induced by length polydispersity but may be brought about by the soft electrostatic interactions only. Moreover, it is shown that the region of manifestation of columnar order can be broadened considerably if the isotropic phase is suppressed. This can be achieved by applying a strong external directional field. Preliminary Brownian dynamics simulations based on a Yukawa-site model are also carried out and the results point to a marked stability of columnar textures at low screening conditions for asymptotically aligned rods, in agreement with theory.

This paper is organized as follows. In Sec. II the general Onsager functional is introduced and the bifurcation analysis is outlined in Sec III. The subsequent sections deal with the explicit calculation of the appropriate Mayer kernels which form the necessary ingredients of the analysis. This is done first for parallel charged rods in Sec. IV and then for freely rotating hard rods (Sec. $\mathrm{V}$ ) and charged ones (Sec. VI). Sec. VII is devoted to the Brownian dynamics simulations and all results will be combined and discussed in Sec. VIII. Finally, some concluding remarks will be formulated in Sec. IX.

\section{ONSAGER FUNCTIONAL}

The starting point of our analysis is to construct a general free energy functional for an inhomogeneous system of $N$ freely rotating rods with arbitrary mutual interactions in a volume $V$. The simplest approach is to take Onsager's well-known result $\underline{1}$ for a virial expansion of the Helmholtz free energy $F$ truncated after the first non-trivial term, which we may recast into the following functional

$$
\begin{aligned}
\beta F[\rho]= & \int \rho(\mathbf{s})\{\ln \mathcal{V} \rho(\mathbf{s})-1\} d \mathbf{s} \\
& -\frac{1}{2} \int d \mathbf{s} \rho(\mathbf{s}) \int d \mathbf{s}^{\prime} \rho\left(\mathbf{s}^{\prime}\right) \Phi\left(\mathbf{s}, \mathbf{s}^{\prime}\right)
\end{aligned}
$$

with $\beta^{-1}=k_{B} T$ the thermal energy ( $k_{B}$ is Boltzmann's constant and $T$ temperature) and $\mathcal{V}$ the thermal volume of a rod. The rod density distribution $\rho$ is a function of the generalized coordinates $\mathbf{s}=\{\mathbf{r}, \Omega\}$, indicating position and solid angle, respectively and is normalized according to $\int d \mathbf{s} \rho(\mathbf{s})=N / V$. The first term in Eq. (11) representing the ideal mixing free energy is exact while the second contribution contains the Mayer function $\Phi\left(\mathbf{s}, \mathbf{s}^{\prime}\right)$ to account for the rod interactions on the approximate pairwise level. The Mayer function is related to the direct rod pair interaction energy $w$ via

$$
\Phi\left(\Delta \mathbf{r} ; \Omega, \Omega^{\prime}\right)=\exp \left[-\beta w\left(\Delta \mathbf{r} ; \Omega, \Omega^{\prime}\right)\right]-1
$$

which depends on the centre-of-mass difference vector $\Delta \mathbf{r}=\mathbf{r}^{\prime}-\mathbf{r}$ of a pair of rods and their orientations. For hard rods $\beta w$ is infinitely large if the particles overlap and zero otherwise. If the system is also homogeneous, the density distribution is independent of position and can be factorized into $\rho=f(\Omega) N / V$, where $f(\Omega)$ is a distribution of orientations obeying $\int d \Omega f(\Omega)=1$. Spatial integration of Eq. (2) then gives the relation

$$
\int d \Delta \mathbf{r} \Phi\left(\Delta \mathbf{r} ; \Omega, \Omega^{\prime}\right)=-v_{\text {excl }}\left(\Omega, \Omega^{\prime}\right)
$$

with $v_{\text {excl }}=2 L^{2} D\left|\sin \gamma\left(\Omega, \Omega^{\prime}\right)\right|$, the excluded volume of two thin hard rods with length $L$ and diameter $D$ (such that $L / D \gg 1$ ) at interrod angle $\gamma$. In general, Eq. (3) forms the basis of the classic Onsager-type theories for homogeneous systems of hard anisometric particles 29 . For any given density $N / V$ the thermodynamics of the system is fully contained in the orientation distribution function $f(\Omega)$ (ODF). Its equilibrium form can be uniquely obtained for a given density by requiring the free energy to be minimal. Formally minimizing the free energy with respect to the ODF leads to the following general stationarity equation for homogeneous systems

$$
f(\Omega)=\mathcal{N}^{-1} \exp \left[\rho \int d \Omega^{\prime} f\left(\Omega^{\prime}\right) \int d \Delta \mathbf{r} \Phi\left(\Delta \mathbf{r} ; \Omega, \Omega^{\prime}\right)\right]
$$

with $\mathcal{N}=\int d \Omega^{\prime} \exp [\cdots]$ to ensure normalization. Upon increasing density, a change of shape of $f(\Omega)$ from a 
constant to a peaked distribution signifies an orientational symmetry-breaking transition of the system from an isotropic state (with random rod orientations) towards e.g. a nematic one where the rods point in globally the same direction, defined as the nematic director.

At higher densities, additional phase transitions from the nematic phase towards states with broken translational symmetry such as smectic or columnar phases can be expected. A convenient way to approximately locate phase transitions of this kind is to apply a bifurcation analysis $^{25,30}$ to the generalized Onsager free energy functional.

\section{BIFURCATION ANALYSIS}

In a smectic or columnar liquid crystal, the density is no longer constant throughout space but shows periodic spatial modulations in one dimension along the nematic director (smectic order) or in a two-dimensional Bravais lattice (hexagonal, cubic, et cetera) perpendicular to the director (columnar order). We may thus propose the following Fourier expansion for the density distribution:

$$
\rho(\mathbf{r}, \Omega)=\rho \sum_{k} \sum_{l \geq 0} a_{l}(\Omega) \exp \left[i l \mathbf{q}_{k} \cdot \mathbf{r}\right]
$$

in terms of the orientation dependent amplitudes $a_{l}(\Omega)$ quantifying positional order along the various Fourier modes related to the wave number $q=2 \pi / \lambda$, with $\lambda$ the typical spacing pertaining to the density modulations. While for smectic order a single wavevector $(k=1)$ suffices, implementing columnar (or crystalline) order requires a superposition of different wave vectors $\left\{\mathbf{q}_{1}, \mathbf{q}_{2}, \cdots\right\}$ to reproduce the desired lattice. We will come back to this later. Identifying $a_{0}(\Omega)=\rho f(\Omega)$ for the homogeneous system, we may simplify the above expansion as follows (omitting the $k$-summation for clarity):

$$
\rho(\mathbf{r}, \Omega)=\rho f(\Omega)\left[1+\sum_{l \geq 1} a_{l} \cos (l \mathbf{q} \cdot \mathbf{r})\right]
$$

where we used that $\mathbf{q}=-\mathbf{q}$. The ODF $f(\Omega)$ is now taken to be that of the nematic reference state for all modes $l$. This means that any coupling between fluctuations in the spatial density and the orientations is neglected. A fully consistent calculation for the nematicsmectic bifurcation 31 shows that the position-orientation coupling is marginally small for strongly aligned, slender rods on which we focus in this study.

Inserting Eq. (6), truncated after $l=1$, into the free energy and expanding the free energy of the new inhomogeneous (I) state with respect to the homogeneous nematic $(\mathrm{N})$ one $\delta F=F_{I}-F_{N}$ up to quadratic order gives the free energy curvature

$$
\delta^{2} \beta F=a_{1}^{2} \rho^{2} \int d \Omega f(\Omega) \int d \Omega^{\prime} f\left(\Omega^{\prime}\right)
$$

$$
\times\left(\frac{\delta\left(\Omega, \Omega^{\prime}\right)}{\rho f(\Omega)}-\hat{\Phi}\left(\mathbf{q} ; \Omega, \Omega^{\prime}\right)\right)
$$

The nematic state becomes locally unstable if the curvature vanishes, $\delta^{2} \beta F=0$. The above expression then simplifies to the bifurcation condition

$$
\rho \int d \Omega f(\Omega) \int d \Omega^{\prime} f\left(\Omega^{\prime}\right) \hat{\Phi}\left(\mathbf{q} ; \Omega, \Omega^{\prime}\right)=1
$$

where the hat denotes a cosine transform of the Mayer function:

$$
\hat{\Phi}\left(\mathbf{q} ; \Omega, \Omega^{\prime}\right)=\int d \Delta \mathbf{r} \Phi\left(\Delta \mathbf{r} ; \Omega, \Omega^{\prime}\right) \cos (\mathbf{q} \cdot \Delta \mathbf{r})
$$

which is the key input of the analysis. The bifurcation density is defined as the smallest non-trivial physical solution $\rho^{*}$ of Eq. (8) with associated wave vector $\mathbf{q}^{*}$. Once the instability has been located, additional information about the thermodynamic stability and the order of the phase transition can be inferred from a parametric expansion about the bifurcation point, as outlined in Ref. 26. In Appendix B we shall reproduce the analysis and derive a general expression for the Landau free energy of the inhomogeneous state with respect to the homogeneous nematic system. This result will then be used to verify the thermodynamic stability of the smectic and columnar states. In the next sections we will derive expressions for $\hat{\Phi}(\mathbf{q})$ for the nematic phase focussing first on parallel charged rods. The theory is then extended to include the effect of rotations.

\section{PARALLEL CHARGED RODS: HARD-CORE YUKAWA SITE MODEL}

To calculate the Mayer kernel for parallel charged rods we have to find a suitable route to include the influence of electrostatic end effects. These end effects are intricately difficult to quantify ${ }^{32}$ but are nevertheless essential in our description since the formation of stable smectic/columnar liquid crystalline structures at high densities is driven primarily by correlations between the end cap of one rod and the main (cylindrical) manifold of the other as embodied in the $\mathcal{O}\left(L D^{2}\right)$ contributions to the Mayer kernel. To make headway we consider a model in which a rod with finite length $L$ is composed of an array of $n \gg 1$ spherical beads with diameter $D$ placed at equidistant intervals. Each bead $i(1 \leq i \leq n)$ from one rod interacts with bead $j$ from the other via a hardcore Yukawa (HCY) potential. The total rod potential depends only on the centre-of-mass distance $\mathbf{s}$ (expressed in units $D$ ) and is given by

$$
\beta w_{e l}^{H C Y}(\mathbf{s})= \begin{cases}\epsilon \sum_{i} \sum_{j} \frac{\exp \left[-\kappa D\left(s_{i j}-1\right)\right]}{s_{i j}} & \text { if all } s_{i j} \geq 1 \\ \infty & \text { if any } s_{i j}<1\end{cases}
$$


with $\kappa$ the Debye screening constant and $\epsilon$ a dimensionless contact potential $\epsilon=(Z / n)^{2}\left(\lambda_{B} / D\right) /(1+\kappa D / 2)^{2}$ depending on the total rod charge $Z$ (in units of the elementary charge $e$ ) and the Bjerrum length $\lambda_{B}=\beta e^{2} / \varepsilon_{0} \varepsilon_{r}$ which is determined by the temperature and the dielectric constant $\varepsilon_{r}$ of the solvent. The quantity $s_{i j}$ represent the distance (in units $D$ ) between site $i$ from one rod and $j$ from the other. It is convenient to introduce cylindrical coordinates, so that $\mathbf{s}=\left\{s_{\perp} \cos \omega, s_{\perp} \sin \omega, s_{\|}\right\}$, with $s_{\perp}$ expressed in units $D$ and $s_{\|}$in units $L$. The site-site distance $s_{i j}$ is then given by

$$
s_{i j}=\sqrt{s_{\perp}^{2}+(L / D)^{2}\left[s_{\|}+d(j-i)\right]^{2}}, \quad i, j=1,2, \cdots n
$$

Here, $d=(L / D) /(\sqrt{n+1} \sqrt{n-1})$ is the dimensionless spacing between two neighboring sites on a rod, chosen such that the quadrupolar moment ${ }^{33}$ of the rod matches that of a homogeneous line charge of length $L$.

The total transformed Mayer function for the HCY-site model is then given by:

$$
\hat{\Phi}_{H C Y}(\mathbf{q})=L D^{2} \int d \mathbf{s} \Phi_{H C Y}(\mathbf{s}) \cos (D \mathbf{q} \cdot \mathbf{s})
$$

The generalized wave vector is given by $\mathbf{q}=\left\{q_{C}, 0, q_{S}\right\}$ in terms of smectic (S) and columnar (C) components. Hence, we can write $D \mathbf{q} \cdot \mathbf{s}=Q_{C} s_{\perp} \cos \omega+Q_{S} s_{\|}$, with the dimensionless wave numbers

$$
Q_{S}=2 \pi\left(\frac{L}{\lambda_{S}}\right), \quad Q_{C}=2 \pi\left(\frac{D}{\lambda_{C}}\right)
$$

related to the lattice spacing $\lambda$.

Integrating over the angle $\omega$ then yields for the electrostatic contribution (denoted by "Y") to the Mayer kernel:

$$
\begin{aligned}
\hat{\Phi}_{Y}\left(Q_{C}, Q_{S}\right)= & 2 \pi L D^{2} \int_{0}^{\infty} 2 d s_{\|} \int_{1}^{\infty} s_{\perp} d s_{\perp} \Phi_{H C Y}\left(s_{\perp}, s_{\|}\right) J_{0}\left(Q_{c} s_{\perp}\right) \cos \left(Q_{S} s_{\|}\right) \\
& +2 \pi L D^{2} \int_{1}^{\infty} 2 d s_{\|} \int_{0}^{1} s_{\perp} d s_{\perp} \Phi_{H C Y}\left(s_{\perp}, s_{\|}\right) J_{0}\left(Q_{c} s_{\perp}\right) \cos \left(Q_{S} s_{\|}\right)
\end{aligned}
$$

where the spatial integration has been performed over the region outside the cylindrical manifold determined by the hard-core excluded volume of two parallel cylinders. The complementary integral over the inner region where the cylindrical cores overlap yields the hard-core contribution (denoted by "H")

$$
\begin{aligned}
\hat{\Phi}_{H}\left(Q_{C}, Q_{S}\right)= & 2 \pi L D^{2} \int_{0}^{1} 2 d s_{\|} \int_{0}^{1} s_{\perp} d s_{\perp} \Phi_{H C Y} \\
& \times J_{0}\left(Q_{c} s_{\perp}\right) \cos Q_{S} s_{\|} \\
= & -4 \pi L D^{2} j_{0}\left(Q_{S}\right) \frac{J_{1}\left(Q_{c}\right)}{Q_{c}}
\end{aligned}
$$

with $j_{0}(x)=\sin x / x$ a spherical Bessel function and $J_{k}$ a standard one. In addition we used that $\Phi_{H C Y}=-1$ for overlapping hard bodies. The result is identical to that of Ref. 26. We remark that the excluded volume between two spherical end caps $\sim \mathcal{O}(D)^{3}$ is not resolved exactly by the integrations of Eqs. (14) and (15) and the Yukawa contribution in Eq. (14) is therefore expected to be reliable up to $\mathcal{O}\left(L D^{2}\right)$, which suffices for slender rods. The integrations in Eq. (14) can be carried out numerically by imposing appropriate cutoff distances for $s_{\perp}$ and $s_{\|}$. The bifurcation condition Eq. (8) is obtained by substituting $\hat{\Phi}=\hat{\Phi}_{H}+\hat{\Phi}_{Y}$ and $f(\Omega)=\delta(\Omega)$.

\section{FREELY ROTATING HARD RODS}

Before embarking on our calculation of $\hat{\Phi}$ for freely rotating charged rods, we will first discuss the effect of rotations on the translational symmetry-breaking bifurcations in systems of hard rods. A closed expression for $\hat{\Phi}(\mathbf{q})$ for freely rotating, hard spherocylinders has been derived by van Roij 34 :

$$
\begin{aligned}
\hat{\Phi}_{H}\left(\mathbf{q} ; \Omega_{1}, \Omega_{2}\right)= & -2 L^{2} D|\sin \gamma| j_{0}(D \mathbf{q} \cdot \hat{\mathbf{v}}) j_{0}\left(\frac{L}{2} \mathbf{q} \cdot \hat{\mathbf{w}}_{\mathbf{1}}\right) j_{0}\left(\frac{L}{2} \mathbf{q} \cdot \hat{\mathbf{w}}_{\mathbf{2}}\right) \\
& -L D^{2} j_{0}\left(\frac{L}{2} \mathbf{q} \cdot \hat{\mathbf{w}}_{\mathbf{1}}\right)\left[2 \pi \cos \left(\frac{L}{2} \mathbf{q} \cdot \hat{\mathbf{w}}_{\mathbf{2}}\right) \frac{J_{1}\left(M_{1}\right)}{M_{1}}+2 \sin \left(\frac{L}{2} \mathbf{q} \cdot \hat{\mathbf{w}}_{\mathbf{2}}\right) W\left(M_{1}, \phi_{1}\right)\right] \\
& -L D^{2} j_{0}\left(\frac{L}{2} \mathbf{q} \cdot \hat{\mathbf{w}}_{\mathbf{2}}\right)\left[2 \pi \cos \left(\frac{L}{2} \mathbf{q} \cdot \hat{\mathbf{w}}_{\mathbf{1}}\right) \frac{J_{1}\left(M_{2}\right)}{M_{2}}-2 \sin \left(\frac{L}{2} \mathbf{q} \cdot \hat{\mathbf{w}}_{\mathbf{1}}\right) W\left(M_{2}, \phi_{2}\right)\right]
\end{aligned}
$$

which contains all contributions up to $\mathcal{O}\left(L D^{2}\right)$. Correction terms of $\mathcal{O}\left(D^{3}\right)$, for which no closed analytical expressions are available, can be safely neglected for suf- 
ficiently anisometric rods $L / D \gg 1$. The inner products contain the rod orientation unit vectors $\hat{\mathbf{w}}_{\mathbf{1}}$ and $\hat{\mathbf{w}}_{\mathbf{2}}$ with associated unit vector $\hat{\mathbf{v}}=\hat{\mathbf{w}}_{\mathbf{1}} \times \hat{\mathbf{w}}_{\mathbf{2}} / \sin \gamma$. The function $W$ is defined as

$$
W(M, \phi)=\int_{-\pi / 2}^{\pi / 2} d \alpha j_{1}(M \cos (\alpha-\phi))
$$

with $j_{1}(x)=x^{-2} \sin x-x^{-1} \cos x$ a spherical Bessel function.

Defining a third unit basis vector $\hat{\mathbf{u}}_{i}=\hat{\mathbf{w}}_{i} \times \hat{\mathbf{v}}$ the quantity $M_{i}$ and the angle $\phi_{i}$ are given by

$$
\begin{aligned}
M_{i} & =D q \sqrt{(\hat{\mathbf{q}} \cdot \hat{\mathbf{v}})^{2}+\left(\hat{\mathbf{q}} \cdot \hat{\mathbf{u}}_{i}\right)^{2}}, \quad i=1,2 \\
\phi_{i} & =\arccos \left(\frac{\hat{\mathbf{q}} \cdot \hat{\mathbf{u}}_{i}}{\sqrt{(\hat{\mathbf{q}} \cdot \hat{\mathbf{v}})^{2}+\left(\hat{\mathbf{q}} \cdot \hat{\mathbf{u}}_{i}\right)^{2}}}\right)
\end{aligned}
$$

The result for perfectly aligned rods is easily recovered by replacing the orthonormal set of basis vectors $\left\{\hat{\mathbf{u}}_{i}, \hat{\mathbf{v}}, \hat{\mathbf{w}}_{i}\right\}$ by the set of Cartesian unit vectors. We may thus identify $\hat{\mathbf{u}}_{1}=\hat{\mathbf{u}}_{2}=\{1,0,0\}, \hat{\mathbf{v}}=\{0,1,0\}$ and $\hat{\mathbf{w}}_{1}=\hat{\mathbf{w}}_{2}=\{0,0,1\}$. With help of the identity $j_{0}(x)=\cos (x / 2) j_{0}(x / 2) \mathrm{Eq}$. (16) then immediately gives back Eq. (15).

Let us now perform an asymptotic analysis of the Mayer kernel, valid for strongly nematic systems where the average deviation of the rod vectors from the nematic director is small.

\section{A. Smectic symmetry}

For the smectic case, density modulations occur along the nematic director $\hat{\mathbf{n}}$ fixed at $\hat{\mathbf{n}}=\{0,0,1\}$ and hence $\mathbf{q}=q_{S}\{0,0,1\}$. The orientation of $\operatorname{rod} i$ on the unit sphere can be parametrized in terms of a polar angle $\theta_{i}$ and an azimuthal one $\varphi_{i}$, so that $\hat{\mathbf{w}}_{i}=$ $\left\{\sin \theta_{i} \sin \varphi_{i}, \sin \theta_{i} \cos \varphi_{i}, \cos \theta_{i}\right\}$. Expanding all relevant inner products for small polar angles $\theta_{i}$ up to leading order gives:

$$
\begin{aligned}
\frac{L}{2} \mathbf{q} \cdot \hat{\mathbf{w}}_{i} & \sim \frac{1}{2} Q_{S} \\
D \mathbf{q} \cdot \hat{\mathbf{v}} & \sim Q_{S} \frac{D}{L}\left(\frac{\theta_{1} \theta_{2}}{\sqrt{\theta_{1}^{2}+\theta_{2}^{2}-2 \theta_{1} \theta_{2} \cos \Delta \varphi}}\right) \\
M_{i} & \sim Q_{S} \frac{D}{L} \theta_{i}
\end{aligned}
$$

with $\Delta \varphi=\varphi_{2}-\varphi_{1}$. For large aspect ratios $(L / D \gg 1)$ all contributions of $\mathcal{O}\left[(D / L) \theta_{i}\right]$ become marginally small so that the following limiting values can be deduced : $M_{i} \rightarrow 0$ and $(D \hat{\mathbf{q}} \cdot \hat{\mathbf{v}}) \rightarrow 0$. Consequently, $J_{1}(M) / M \rightarrow$ $1 / 2$ and $W(M, \phi) \rightarrow 0$. Using this in Eq. (16) together with the identity $j_{0}(x)=\cos (x / 2) j_{0}(x / 2)$ gives the following asymptotic expression for the transformed Mayer function for the smectic symmetry:

$$
\begin{aligned}
\hat{\Phi}_{H}\left(\mathbf{q} ; \Omega_{1}, \Omega_{2}\right)= & -2 L^{2} D\left|\sin \gamma\left(\Omega_{1}, \Omega_{2}\right)\right| j_{0}^{2}\left(Q_{S} / 2\right)- \\
& 2 \pi L D^{2} j_{0}\left(Q_{S}\right)
\end{aligned}
$$

The latter contribution is simply the result for parallel rods, given by Eq. (15) taking the limit $Q_{c} \rightarrow 0$, while the first is the leading order correction term arising from the rotations.

The bifurcation condition is obtained by performing an orientational average according to Eq. (8). Following Odijk ${ }^{35}$, we introduce the Gaussian trial ODF

$$
f_{G}(\theta) \cong \frac{\alpha}{4 \pi} \begin{cases}\exp \left[-\frac{1}{2} \alpha \theta^{2}\right] & \text { if } 0 \leq \theta \leq \frac{\pi}{2} \\ \left.\exp \left[-\frac{1}{2} \alpha(\pi-\theta)\right)^{2}\right] & \text { if } \frac{\pi}{2}<\theta \leq \pi\end{cases}
$$

which depends only on the polar angle in case of uniaxial nematic order. The variational parameter $\alpha$ is chosen such as to minimize the nematic free energy. It must be much larger than unity because the normalization factor is only valid in this limit. After minimization $\alpha$ attains a quadratic density dependence, $\alpha=4 c^{2} / \pi$ where $c$ is a dimensionless rod concentration $c=(\pi / 4) \rho L^{2} D$ related to the volume fraction $\eta$ via $c=\eta L / D$. We may now use the following asymptotic result $\stackrel{1}{ }$ :

$$
\langle\langle|\sin \gamma|\rangle\rangle_{f_{G}} \sim\left(\frac{\pi}{\alpha}\right)^{1 / 2}
$$

where the brackets denote an orientational average. With this, the bifurcation equation Eq. (8) finally becomes

$$
-4 j_{0}^{2}\left(Q_{S} / 2\right)-8 \eta j_{0}\left(Q_{S}\right)=1
$$

Note that the rotational correction term is independent of the density.

\section{B. Columnar symmetry}

Let us now turn to the columnar state where a twodimensional modulatory density pattern $\perp \hat{\mathbf{n}}$ is expected. In case of a hexagonal Bravais lattice, a combination of the following three unit wavevectors:

$$
\hat{\mathbf{q}}_{1}=\left(\begin{array}{l}
0 \\
1 \\
0
\end{array}\right), \quad \hat{\mathbf{q}}_{2}=\left(\begin{array}{c}
\sqrt{3} / 2 \\
1 / 2 \\
0
\end{array}\right), \quad \hat{\mathbf{q}}_{3}=\left(\begin{array}{c}
-\sqrt{3} / 2 \\
1 / 2 \\
0
\end{array}\right)
$$

correctly reproduces the six-fold symmetry of the columnar density modulations in the $x y$ plane. We may now repeat the asymptotic analysis to obtain the leading order expressions for the inner products. For the $\mathbf{q}_{1}$-mode we obtain

$$
\begin{aligned}
\frac{L}{2} \mathbf{q}_{1} \cdot \hat{\mathbf{w}}_{i} & \sim \frac{1}{2} Q_{C} \frac{L}{D} \theta_{i} \sin \varphi_{i} \\
D \mathbf{q}_{1} \cdot \hat{\mathbf{v}} & \sim Q_{C}\left(\frac{-\theta_{1} \cos \varphi_{1}+\theta_{2} \cos \varphi_{2}}{\sqrt{\theta_{1}^{2}+\theta_{2}^{2}-2 \theta_{1} \theta_{2} \cos \Delta \varphi}}\right) \\
M_{i} & \sim Q_{C}
\end{aligned}
$$

The expressions for the other columnar modes show exactly the same scaling with respect to $\theta$ and $L / D$ and 
are not shown. A quick inspection reveals that the asymptotic analysis does not bring much relief since i) $(\hat{\mathbf{q}} \cdot \hat{\mathbf{v}}) \sim \mathcal{O}(1)$ and ii) the combination $(L / D) \theta_{i}$ appearing in the first line also remains finite after having performed the orientational average. It can be estimated with use of the Gaussian ODF Eq. (21) and the resulting scaling relation reads $\langle(L / D)|\theta|\rangle_{f_{G}} \sim \eta^{-1} \sqrt{\pi / 2} \sim \mathcal{O}(1)$.

Unlike for the smectic case, the complicated nature of the orientation dependent terms precludes an analytical calculation of their Gaussian averages. Nevertheless, the integration over the angles $\theta_{i}$ and $\varphi_{i}$ can be carried out numerically without difficulty using standard Simpson quadrature ${ }^{36}$. The bifurcation condition Eq. (8) for the columnar symmetry is given by a linear superposition of kernels

$$
\frac{\rho}{3} \sum_{k=1}^{3}\left\langle\left\langle\hat{\Phi}_{H}\left(\mathbf{q}_{k} ; \theta_{1}, \varphi_{1} ; \theta_{2}, \varphi_{2}\right)\right\rangle\right\rangle_{f_{G}}=1
$$

substituting the asymptotic forms Eq. (25) in Eq. (16) and using the Gaussian ODF Eq. (21).

\section{Bifurcation points}

The solutions of Eqs. (23) and (26) have been collected in Table I. To obtain more realistic volume fractions a density rescaling according to Parsons-Lee (PL) method $37,38,39$ has been carried out. The approach, not treated here, can be implemented quite simply by rescaling the density according to $\rho \rightarrow \rho g_{C S}(\eta)$, and equivalently $\eta \rightarrow \eta g_{C S}(\eta)$, where $g_{C S}(\eta)=(1-(3 / 4) \eta) /(1-\eta)^{2}$ originates from the Carnahan-Starling equation of state for a hard sphere fluid. All volume fractions mentioned throughout the rest of the paper are obtained from this treatment.

It is clear that both N-S and N-C are destabilized due to the effect of rotations. The decrease of the smectic layer spacing can be intuitively understood from the fact that the rod length projected onto the nematic director (the 'entropic' length) is smaller than the bare rod length due to the orientational fluctuations. Similarly, the increased columnar spacing can be attributed to an entropic rod diameter larger then the bare one. The N-S pre-empting the N-C one is in accordance with simulations ${ }^{27.40}$ and experimental observations $\frac{17}{17}$. In addition, the location of the nematic-smectic transition predicted here compares very well with the value $\eta_{S}^{*} \approx 0.418$ reported from simulations ${ }^{3}$.

\section{FREELY ROTATING CHARGED RODS}

Looking back at the expressions derived for hard rods, in particular Eq. (20), we may conclude that the asymptotic Mayer kernel consist of two parts. First, a 'reference' part for (near)-parallel configurations associated

\begin{tabular}{|l||l|l|l|l|}
\hline & $\eta_{S}^{*}$ & $Q_{S}^{*}$ & $\eta_{C}^{*}$ & $Q_{C}^{*}$ \\
\hline parallel rods & 0.575 & 4.493 & 0.945 & 5.136 \\
\hline parallel rods (PL) & 0.338 & 4.493 & 0.441 & 5.136 \\
\hline rotating rods & 0.792 & 4.858 & 1.540 & 4.424 \\
\hline rotating rods (PL) & 0.404 & 4.858 & 0.543 & 4.424 \\
\hline
\end{tabular}

TABLE I: Overview of the nematic-smectic and nematiccolumnar bifurcations for hard rods. (PL) refers to ParsonsLee theory.

with the $\mathcal{O}\left(L D^{2}\right)$ contributions in Eq. (16)) and, second, a part of $\mathcal{O}\left(L^{2} D\right)$ which constitutes a non-vanishing contribution in the limit of asymptotically strong alignment. In this section we will first derive the leading order $\mathcal{O}\left(L^{2} D\right)$ correction due to electrostatic effects. These correction terms will then, together with previous results, be compiled into expressions for the total Mayer kernel for charged rods in near-parallel configurations.

Following Ref. 21 we start with introducing the following form for the electrostatic interaction between two infinitely long charged rods at shortest distance $x$ and mutual angle $\gamma$ :

$$
\beta w_{e l}\left(x ; \Omega_{1}, \Omega_{2}\right)=A \frac{\exp [-\kappa(|\mathbf{x}|-D)]}{\left|\sin \gamma\left(\Omega_{1}, \Omega_{2}\right)\right|}
$$

If the rod charge is not too high, the prefactor $A$ can be expressed in closed form within the Debye-Hückel approximation

$$
A=\frac{8 \pi\left(\nu \lambda_{B}\right)^{2} \exp [-\kappa D]}{(\kappa D)^{3}\left(\lambda_{B} / D\right) K_{1}^{2}(\kappa D / 2)}
$$

with $K_{1}(x)$ a modified Bessel function. This expression stems from the linearized Poisson-Boltzmann equation for an infinitely long, charged cylinder with diameter $D$. The key quantities here are the linear charge density $\lambda_{B} \nu$ expressed in terms of unit charges per Bjrerrum length and the ratio of the electric double layer thickness and the rod diameter $1 / \kappa D$. The denominator in Eq. (27) embodies the electrostatic 'twisting' effect whereby a rod pair is energetically stimulated to adopt a perpendicular configuration. Clearly, for strictly parallel configurations, the expression is ill-defined which means that a N-I bifurcation analysis cannot be based solely on this potential.

The electrostatic line charge contribution of the cosine transform of the Mayer kernel can now be written as

$$
\begin{aligned}
\hat{\Phi}_{e l}\left(\mathbf{q} ; \Omega_{1}, \Omega_{2}\right)= & \int_{\substack{|\mathbf{x}|>D\\
}} d|\mathbf{x}|\left\{\exp \left[-\beta w_{e l}\left(|\mathbf{x}| ; \Omega_{1}, \Omega_{2}\right)\right]-1\right\} \\
& \times \cos (\mathbf{q} \cdot \mathbf{x})
\end{aligned}
$$

If we neglect end effects, the shortest distance unit vector $\hat{\mathbf{x}}$ is equal to $\hat{\mathbf{v}}$ and the following parametrization $\mathbf{x}=$ $D t \hat{\mathbf{v}}$, with $1<t<\infty$, can be applied. Rewriting the 
integral gives

$$
\begin{aligned}
\hat{\Phi}_{e l}= & D \int_{1}^{\infty} d t\left\{\exp \left[-\left(A^{\prime} /|\sin \gamma|\right) e^{-\kappa D t}\right]-1\right\} \\
& \times \cos (t D \mathbf{q} \cdot \hat{\mathbf{v}})
\end{aligned}
$$

with $A^{\prime}=A \exp [\kappa D]$. To solve the integral it is advantageous to make the following substitution $u(t)=$ $\left(A^{\prime} /|\sin \gamma|\right) \exp [-\kappa D t]$ and to recast it into a complete Fourier integral (denoted by the tilde). This gives:

$$
\kappa \tilde{\Phi}_{e l}=\int_{0}^{u_{1}} d u u^{-1}\left(e^{-u}-1\right) \exp \left[-i \tilde{q} \ln \left(u / u_{0}\right)\right]
$$

with $u_{m}=u(m)$ and $\tilde{q}=\kappa^{-1} \mathbf{q} \cdot \hat{\mathbf{v}}$. The complex solution of the integral reads

$$
\kappa \tilde{\Phi}_{e l}=-u_{0}^{i \tilde{q}}\left(\frac{i u_{1}^{-i \tilde{q}}}{\tilde{q}}+\Gamma\left(-i \tilde{q}, u_{1}\right)-\Gamma(-i \tilde{q})\right)
$$

in terms of the complete and incomplete gamma functions, $\Gamma(z)$ and $\Gamma(a, z)$ respectively. Because of the cosine transform in Eq. (30) we only need the real part $\operatorname{Re}\left(\kappa \tilde{\Phi}_{e l}\right)$. In the asymptotic limit, $u_{1}=A /|\sin \gamma| \gg 1$ and the incomplete gamma function becomes negligibly small so that it can be omitted in the remainder of the analysis. Writing out the complex functions one arrives at

$$
\kappa \hat{\Phi}_{e l} \approx \frac{\sin \tilde{q} \kappa D}{\tilde{q}}+\operatorname{Re}\left[u_{0}^{-i \tilde{q}} \Gamma(i \tilde{q})\right]
$$

To further approximate the latter term let us first note that for the smectic and columnar modes we have

$$
\begin{aligned}
& \tilde{q}_{S}=(\kappa L)^{-1} Q_{S}(\hat{\mathbf{q}} \cdot \hat{\mathbf{v}}) \ll 1 \\
& \tilde{q}_{C}=(\kappa D)^{-1} Q_{C}(\hat{\mathbf{q}} \cdot \hat{\mathbf{v}}) \sim \mathcal{O}(1)
\end{aligned}
$$

recalling that $\kappa D \sim \mathcal{O}(1)$ and $L / D \gg 1$. For the smectic symmetry, we may approximate

$$
-\Gamma(i \tilde{q}) \approx \gamma_{E}+i \tilde{q}^{-1}, \quad \tilde{q} \ll 1
$$

with $\gamma_{E}$ Euler's constant to obtain:

$$
\begin{aligned}
\hat{\Phi}_{e l}= & D j_{0}\left(\kappa D \tilde{q}_{S}\right) \\
& -\kappa^{-1}\left\{\frac{\sin \left(\kappa \bar{D} \tilde{q}_{S}\right)}{\tilde{q}_{S}}-\gamma_{E} \cos \left(\kappa \bar{D} \tilde{q}_{S}\right)\right\}
\end{aligned}
$$

with $\bar{D}=D\left\{1+(\kappa D)^{-1}(\ln A-\ln |\sin \gamma|)\right\}$. It is easy to verify that the electrostatic contribution vanishes in the hard rod limit $(\kappa D \rightarrow \infty)$ as it should. Taking the limit $\tilde{q}_{S} \rightarrow 0$ Eq. (36) simplifies to:

$$
\hat{\Phi}_{e l}=-\kappa^{-1}\left(\ln A-\ln |\sin \gamma|+\gamma_{E}\right)
$$

We may now compose the total Mayer kernel by replacing the thickness contribution $j_{0}(D \mathbf{q} \cdot \hat{\mathbf{v}})$ in Eq. (16) by
Eq. (37). The contributions depending on $L$ are left untouched since the line charge model, by definition, can only affect the interaction thickness of the rod. These considerations lead to the following form of the Mayer kernel of two line charges:

$$
\hat{\Phi}\left(\mathbf{q} ; \Omega_{1}, \Omega_{2}\right)=-2 L^{2} D_{\text {eff }}\left(\Omega_{1}, \Omega_{2}\right)|\sin \gamma| j_{0}^{2}\left(Q_{S} / 2\right)
$$

valid for the smectic symmetry. It is similar to the first term in Eq. (20) but with $D$ replaced by an orientationdependent effective thickness $D_{\text {eff: }}$ :

$$
D_{\text {eff }}\left(\Omega_{1}, \Omega_{2}\right)=D\left\{1+\frac{1}{\kappa D}\left(\ln A-\ln |\sin \gamma|+\gamma_{E}\right)\right\}
$$

which is exactly the same as the effective thickness showing up in the second virial coefficient of two charged rods in Ref. 21.

The next step is to perform the orientational average using Eq. (21). The variational parameter $\alpha$ now follows from minimizing the nematic free energy for charged rods. The associated minimum condition reads 21

$$
\alpha-\mathcal{K}_{1} c \alpha^{1 / 2}+\mathcal{K}_{2} c \alpha^{1 / 2}(2-\ln \alpha)=0
$$

with constants $\mathcal{K}_{1}=\pi^{-1 / 2}\left[2+(\kappa D)^{-1}\left(2 \ln A+3 \gamma_{E}-2\right)\right]$ and $\mathcal{K}_{2}=\pi^{-1 / 2}(\kappa D)^{-1}$. This equation has to be solved numerically for any given concentration $c$ and electrostatic parameters $A$ and $\kappa D$. One may verify the hard rod limit $\kappa D \rightarrow \infty$ to obtain $\alpha_{H}=4 c^{2} / \pi$. The orientation average of Eq. (38) can be worked with the aid of Eq. (22) and the following asymptotic result 21

$$
\langle\langle|\sin \gamma| \ln |\sin \gamma|\rangle\rangle_{f_{G}} \sim\left(\frac{\pi}{4 \alpha}\right)^{1 / 2}\left(\ln \alpha-2+\gamma_{E}\right)
$$

It is expedient to normalize the variational parameter $\tilde{\alpha}=\alpha / \alpha_{H}$, so that $\tilde{\alpha}$ approaches unity in the hard $\operatorname{rod}$ limit. Using this, the nematic-smectic bifurcation condition Eq. (8) for charged rods finally becomes

$$
\begin{aligned}
1= & -4 j_{0}^{2}\left(Q_{S} / 2\right) \tilde{\alpha}^{-1 / 2} \\
& \times\left\{1+\frac{1}{\kappa D}\left(\ln A+\frac{1}{2} \ln \alpha+\frac{3}{2} \gamma_{E}-1\right)\right\} \\
& -8 \eta j_{0}\left(Q_{S}\right)+\rho \hat{\Phi}_{Y}\left(Q_{S}, 0\right)
\end{aligned}
$$

with $\hat{\Phi}_{Y}$ given by Eq. (14). Comparing this with the hard rod result Eq. (23) we see that the first contribution is now implicitly dependent on concentration, albeit weakly, due to the nonlinear character of Eq. (40).

For the columnar symmetry we have to retain the complex gamma functions in Eq. (33) and the resulting expression reads:

$$
\begin{aligned}
\hat{\Phi}_{e l}= & D j_{0}\left(\kappa D \tilde{q}_{C}\right)+\kappa^{-1}\left\{\cos \left(\kappa \bar{D} \tilde{q}_{C}\right) \operatorname{Re}\left[\Gamma\left(i \tilde{q}_{C}\right)\right]\right. \\
& \left.+\sin \left(\kappa \bar{D} \tilde{q}_{C}\right) \operatorname{Im}\left[\Gamma\left(i \tilde{q}_{C}\right)\right]\right\}
\end{aligned}
$$

With the aid of Eq. (35), it is easily shown that the contribution vanishes in the limit $\kappa D \rightarrow \infty$ (corresponding to $\left.\tilde{q}_{C} \rightarrow 0\right)$. 
We may combine this with the remaining contributions that depend only on the rod length $L$. The Mayer kernel of $\mathcal{O}\left(L^{2} D\right)$ for two line charges within the columnar symmetry can then be written in the following form:

$$
\begin{aligned}
\hat{\Phi}= & -2 L^{2} D|\sin \gamma| j_{0}\left(\frac{L}{2} \mathbf{q} \cdot \hat{\mathbf{w}}_{\mathbf{1}}\right) j_{0}\left(\frac{L}{2} \mathbf{q} \cdot \hat{\mathbf{w}}_{\mathbf{2}}\right) \\
& \times G\left(\mathbf{q} ; \Omega_{1}, \Omega_{2}\right)
\end{aligned}
$$

where $G$ now replaces the Bessel function $j_{0}(D \mathbf{q} \cdot \hat{\mathbf{v}})$ in Eq. (16)

$$
\begin{aligned}
G\left(\mathbf{q} ; \Omega_{1}, \Omega_{2}\right)= & -(\kappa D)^{-1}\left\{\cos \left(\kappa \bar{D} \tilde{q}_{C}\right) \operatorname{Re}\left[\Gamma\left(i \tilde{q}_{C}\right)\right]\right. \\
& \left.+\sin \left(\kappa \bar{D} \tilde{q}_{C}\right) \operatorname{Im}\left[\Gamma\left(i \tilde{q}_{C}\right)\right]\right\}
\end{aligned}
$$

and the reader may verify that this contribution is recovered from $G$ in the hard rod limit, as it should. With Eq. (14) the total Mayer kernel for the columnar symmetry is proposed to be of the following form:

$$
\hat{\Phi}=\hat{\Phi}_{L^{2} D}\left(\mathbf{q} ; \Omega_{1}, \Omega_{2}\right)+\hat{\Phi}_{L D^{2}}\left(\mathbf{q} ; \Omega_{1}, \Omega_{2}\right)+\hat{\Phi}_{Y}\left(0, Q_{c}\right)
$$

with $\hat{\Phi}_{L^{2} D}$ given by Eq. (44) and $\hat{\Phi}_{L D^{2}}$ the hard-core contribution of $\mathcal{O}\left(L D^{2}\right)$ from Eq. (16), both depending intricately on the rod orientations. Since the latter contribution no longer pertains to the excluded volume of parallel rods, as was the case for the smectic symmetry, the leading order term from the Yukawa site model $\Phi_{Y}$ is expected to be orientation dependent too. However, getting access to this contribution requires a full numerical integration over all spatial and orientational variables of the HCY site model for freely rotating rod pairs. Resolving the Mayer kernel would then give a 9 dimensional integration (including the site-site summation) which clearly is a formidable numerical task. Therefore, we shall rely on the parallel contribution. The approximation can be justified in part from the fact that the variational parameter $\alpha$ and hence the degree of nematic order becomes rather large for sufficiently large Debye lengths, as we will see later on. Finally, the nematiccolumnar bifurcations can be computed by inserting the full Mayer kernel into the bifurcation condition Eq. (26) and performing a numerical averaging over the orientational degrees of freedom using the Gaussian ODF.

\section{BROWNIAN DYNAMICS SIMULATIONS}

To supplement the theory, Brownian dynamics (BD) simulations have been carried out for a system of point Yukawa-site rods. The corresponding interrod potential is virtually identical to Eq. (10) with omission of the hardcore contribution for $s_{i j}<1$ (achieved by taking the limit $D \rightarrow 0$ ). This means that overlaps of the inner cylindrical cores are in principle allowed in our simulations. However these configurations are rare because of the significant energy penalty involved. Another difference with the description in Sec. IV is that the rods are no longer fixed in parallel configurations but are allowed to rotate freely. Apart from the rod length and the Bjerrum length, the relevant lengthscale of the point Yukawa model is the Debye length. The ratio of the latter two can be expressed as

$$
\kappa \lambda_{B}=\sqrt{4 \pi Z \tilde{\rho}\left(\lambda_{B} / L\right)^{3}}
$$

in terms of the dimensionless rod concentration $\tilde{\rho}=$ $N L^{3} / V$.

The simulations comprise a finite difference integration of the Langevin equations for interacting Brownian macro-ions according to the scheme of Ermak ${ }^{41}$. For a detailed exposition of the update equations for Yukawasite rods the reader is referred to the paper of Kirchhoff et al $l^{33}$. The short-time self-diffusive behavior of the rods is characterized by two translational diffusion coefficients (one parallel and perpendicular to the rod axis) and a rotational one. All of these depend on the hydrodynamic aspect ratio of the rods 42 for which we take a value of 16 , comparable to that of TMV rods 17 .

Systems consisting of $N=500$ rods each with $n=13$ sites were simulated in a cubic box with periodic boundary conditions. The dimensionless concentration was fixed at a low value $\tilde{\rho}=4.0$. Starting configurations were generated by randomly inserting rods in a parallel, non-overlapping configuration. Equilibration is then carried out until the system has reached a stable isotropic configuration, characterized by a vanishing nematic order parameter, defined as the maximum eigenvalue of the tensor

$$
\mathbf{Q}=\frac{1}{N} \sum_{i} \frac{3}{2}\left\langle\hat{\mathbf{w}}_{i} \otimes \hat{\mathbf{w}}_{i}\right\rangle-\frac{1}{2} \mathbf{I}
$$

where $\otimes$ denotes a dyadic product, I the second-rank unit tensor and the brackets a canonical average. The next step in our simulations is to nematize the system by applying an external directional field along the $z$-direction of the simulation box. This gives rise to an external potential energy per rod, given by

$$
\beta U_{i}=-\xi \cos ^{2}\left(\hat{\mathbf{w}}_{i} \cdot \hat{\mathbf{z}}\right)
$$

in terms of a dimensionless field strength $\xi>0$ (in units $\left.k_{B} T\right)$. Obviously, a stable nematic state could also have been obtained by increasing the density of the system. However, we found that the simulations become increasingly cumbersome for large densities due to the slow dynamics and the formation of metastable, transient states. Moreover, at high packing fractions the results are expected to be sensitive to the details of the model (in particular the number of sites per rod) and many timeexpensive test runs need to be carried out.

Once the directional field is switched on, the (instantaneous) nematic order parameter is found to increase rapidly until a plateau value is reached after some time interval. The associated average nematic order parameter is found to be close to unity for $\xi=50$ and $\xi=25$ 

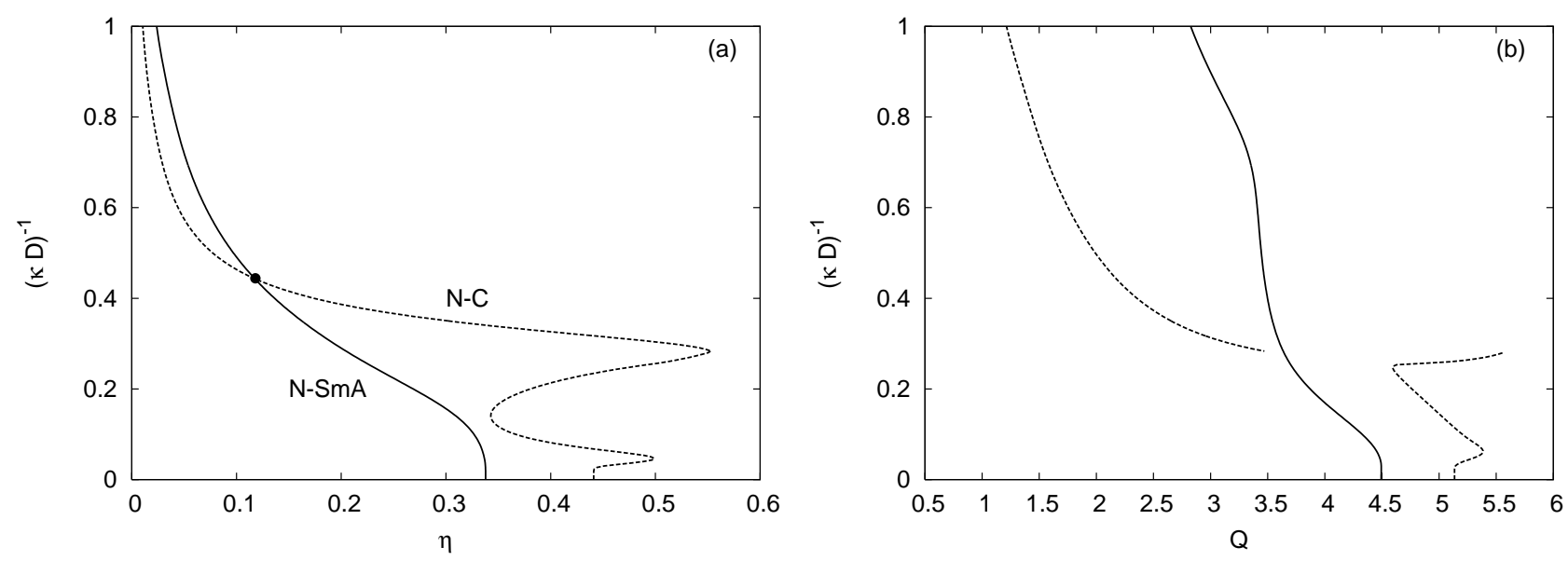

FIG. 1: (a) Bifurcation diagram for parallel charged rods. Plotted is the normalized Debye length $\kappa^{-1} / D$ versus the hardcore volume fraction $\eta$. At $(\kappa D)^{-1}>0.444$ a stable nematic-columnar bifurcation pre-empts the nematic-smectic one. The volume fractions are obtained from Parsons rescaling. (b) Dimensionless wave number $Q$ [Eq. (13)] corresponding to the bifurcation lines shown in (a).

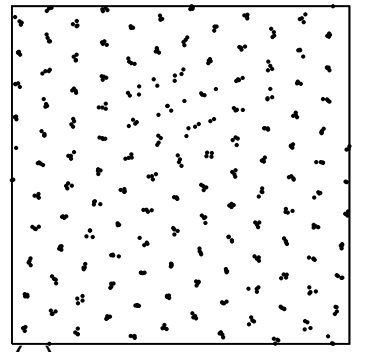

(a)

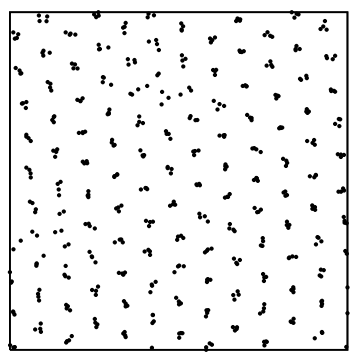

(b)
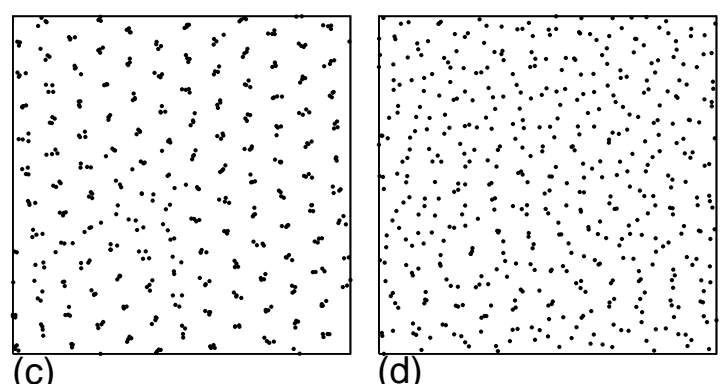

(d)

FIG. 2: Simulation snapshots showing the projections of the rod centres-of mass (indicated by dots) onto the $x y$-plane perpendicular to the field direction. Results correspond to various values for the external field strength $\xi$ [Eq. [49)]. (a) parallel rods $(\xi \rightarrow \infty)$, (b) freely rotating rods at $\xi=50$, (c) same for $\xi=25$ and (d) $\xi=10$.

(near parallel systems) and around 0.8 for the lowest nonzero applied field strength $\xi=10$. The nematic state is then equilibrated further to allow for possible translational freezing transitions. To detect smectic order (along $\hat{\mathbf{z}}$ ) or columnar order (perpendicular to $\hat{\mathbf{z}}$ ) we first intro- duce the intralayer pair correlation function, defined as $\underline{43}$

$$
g_{l}(r)=\frac{1}{N \rho}\left\langle\sum_{i} \sum_{j \neq i} \delta\left(r-\left|\mathbf{r}_{i j} \times \hat{\mathbf{z}}\right|\right) \Theta\left(L / 2-\mathbf{r}_{i j} \cdot \hat{\mathbf{z}}\right)\right\rangle
$$

with $\delta$ the Dirac delta function and $\Theta$ the Heaviside step function. It represents the probability to find a particle at distance $r$ from a reference particle within a slab of thickness $L$ perpendicular to the director. Basically, $g_{l}$ provides information about positional order perpendicular to the nematic director and a profoundly peaked function is to be expected in case of a columnar or crystal structure. Second, the parallel pair correlation function, given by

$$
g_{p}(r)=\frac{1}{N \rho}\left\langle\sum_{i} \sum_{j \neq i} \delta\left(r-\mathbf{r}_{i j} \cdot \hat{\mathbf{z}}\right)\right\rangle
$$

monitors liquid structure parallel to the nematic director and a peaked $g_{p}$ will appear in case of smectic or crystalline order. To make sure the results do not suffer from finite size effects, additional simulations have been carried out using $N=900$ rods with $n=13$ and no qualitative differences were observed. Furthermore, the pressure tensor $\underline{44}$ has been monitored in all cases and no evidence for spurious, non-isotropic stresses induced by the cubic periodic boundary conditions was encountered.

\section{RESULTS AND DISCUSSION}

Before discussing the results we first have to specify the electrostatic parameters of the rod solution. For these we take typical values for TMV rods suspended in water $\left(\varepsilon_{r}=78\right)$ at neutral $\mathrm{pH}$ and room temperature. The rod dimensions are $D=18 \mathrm{~nm}$ and $L=300 \mathrm{~nm}$ and 

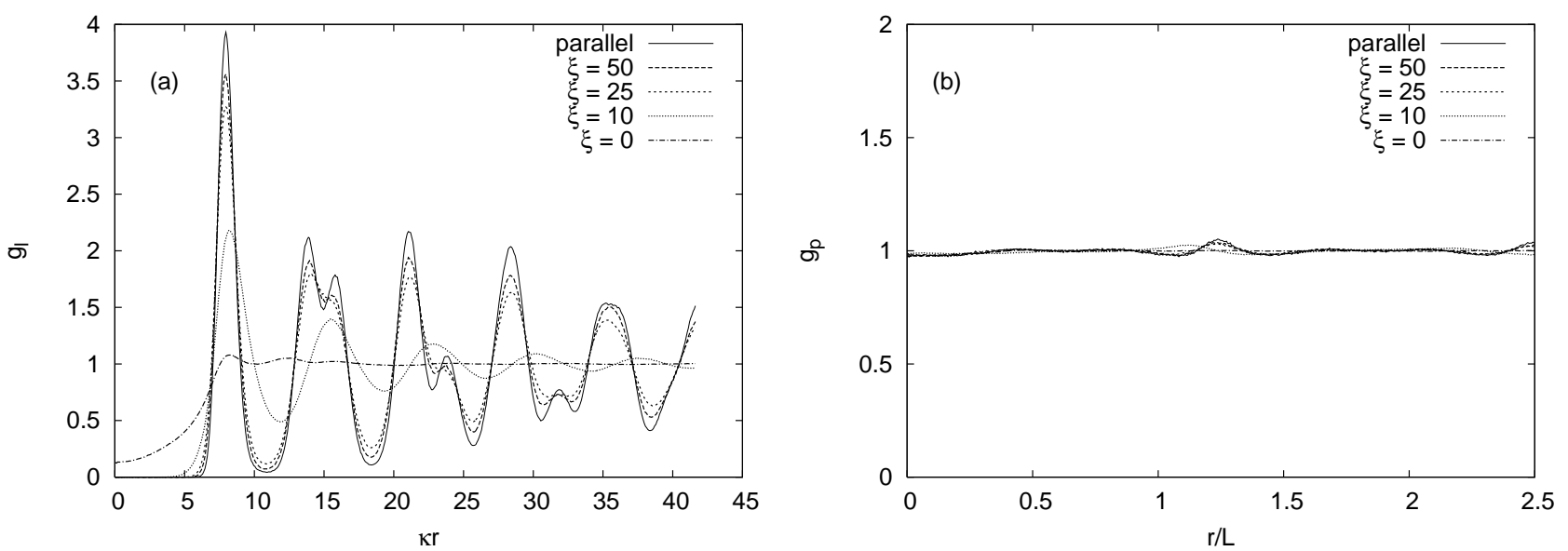

FIG. 3: (a) The intralayer and (b) the parallel pair correlation functions for various field strengths $\xi$.

the Bjerrrum length is $\lambda_{B}=0.716 \mathrm{~nm}$. The total rod charge is fixed at $Z=390$ and the corresponding linear charge density is set at $\lambda_{B} \nu=1$ (i.e. one unit charge per Bjerrum length) which are reasonable values if the effect of counterion condensation is taken into account 10.17 . These parameters justify the use of the Debye-Hückel approximation to determine the potential amplitude in Eq. $(28)^{45}$.

In Fig. 1 results are presented for parallel charged rods with $n=30 \mathrm{HCY}$ sites. Increasing the number of sites per rod did not lead to significant changes in the bifurcation diagram. At high ionic strengths $\left[(\kappa D)^{-1} \leq 0.1\right]$ the rod charges are highly screened and the phase behavior is virtually unaffected by the charge. A stable nematic-smectic bifurcation pre-empts the nematiccolumnar one. At lower ionic strength the transition shifts to lower volume fraction due to fact that the enhanced pair interactions lead to an effective 'interaction' volume fraction higher than the bare one. A qualitative change of scenario occurs at even lower ionic strengths $\left[(\kappa D)^{-1} \geq 0.44\right]$ where a nematic-columnar instability pre-empts the nematic-smectic one and a stable columnar phase can be expected. In this regime, the extension of the double layers is significant and very little packing is needed for the system to order. The generic stabilisation of positional order is in accordance with the results of Ref. 22 and 23. The enhanced interaction range also shows up in the bifurcation wave numbers in Fig. 1b where the sharp decrease implies a columnar spacing spanning multiple rod diameters. The jump at $(\kappa D)^{-1} \approx 0.28$ is a consequence of the fact that there are two independent bifurcating solutions (i.e. a high- $Q$ branch and a low- $Q$ one) whose densities merge at that point.

The stability of the columnar state is confirmed by the simulation results for the parallel Yukawa site model. The snapshot in Fig. 2a displays a hexagonal pattern indicative of freezing perpendicular to the nematic director. The columnar nature of the structure is reflected explic- itly in the pair correlation functions shown in Fig. 3. As to $g_{l}$, the height of the first peak and the double-peaked shape of the second are a hallmark of long-ranged positional order. More importantly, the flatness of $g_{p}$ signals an absence of long-range structure along the director (the small peak is due to weak intra-columnar correlations). This means that there is no evidence of additional layering in the $z$-direction indicative of three-dimensional crystalline order. To roughly estimate whether the state point adopted in the simulations corresponds to the bifurcation diagram in Fig. 1 we may take the hydrodynamic aspect ratio $x_{h}=16.7$ and thickness to estimate $(\kappa D)^{-1} \sim 2.4$ and $\eta \sim(\pi / 4) \tilde{\rho} x_{h}^{2}=0.011$. Although the screening length is beyond the scale depicted in Fig. 1 it can be easily inferred by extrapolation that the state point falls roughly in the columnar stability regime.

Let us now turn to the case of freely rotating rods. The main question is whether or not the scenario sketched above is altered qualitatively if rotational degrees of freedom are allowed for. The corresponding bifurcation diagram in Fig. 4 shows that this is not the case. In fact, the pre-emption of the nematic-columnar seems more outspoken here: the crossover point has shifted to lower values and the the difference between the $\mathrm{N}-\mathrm{S}$ and $\mathrm{N}$ $\mathrm{C}$ volume fraction is enhanced beyond the intersection point. However, in order to be able to make a final assessment of the stability of the inhomogeneous phases we have to verify the stability of the nematic state itself. To illustrate this, the bifurcation from the isotropic to the nematic state has been included in Fig. 4. This curve is obtained from a simple analysis described in Appendix A. Below the I-N bifurcation, the nematic phase is no longer stable with respect to the isotropic and all instabilities of the nematic state located in this region become meaningless. For this reason, the scope of the bifurcation diagram is bounded by the intersection point at $(\kappa D)^{-1} \approx 0.4$. Below this point, the overall scenario is a nematic-smectic transition occurring at high screening $(\kappa D)^{-1}<0.27$ while a gradual crossover towards colum- 


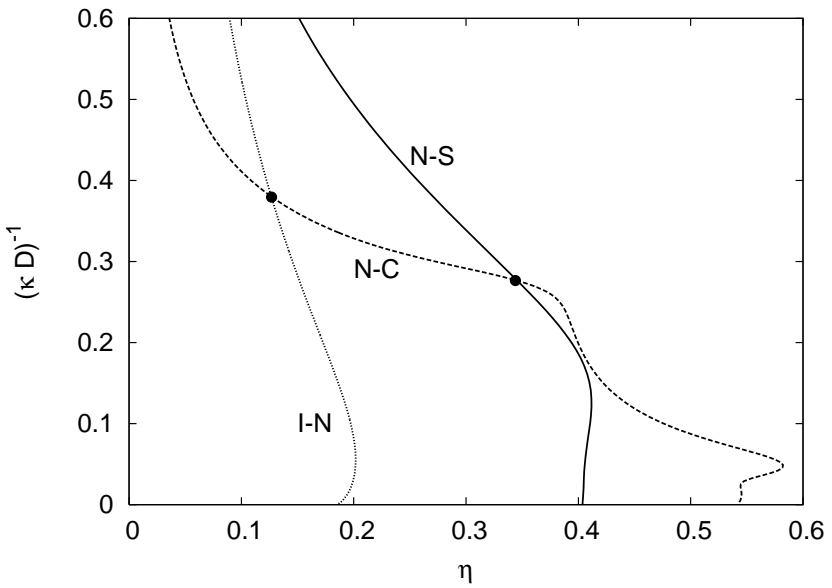

FIG. 4: Bifurcation diagram for freely rotating charged rods. Plotted is the normalized Debye length $\kappa^{-1} / D$ versus the hardcore volume fraction $\eta$. The isotropic-nematic (I-N) bifurcation is obtained from Eq. (56) in Appendix A.

nar order is expected at low salt conditions. The regime of low screening [i.e. $(\kappa D)^{-1}>0.4$ ], cannot be accessed but it is reasonable to anticipate translational symmetrybreaking instabilities (of either columnar or crystalline signature) of the isotropic phase. These are beyond the scope of the present calculations. The Gaussian variational parameter at the instability is recorded in Fig. 5 and its large value (the minimum is at $\alpha \approx 50$ ) supports the use of the asymptotic analysis adopted throughout this paper.

If the isotropic state were to be suppressed by applying a strong aligning external field, the nematic phase will be stable irrespective of density and pronounced columnar order can be expected in a large portion of the phase diagram, as we see in Fig. 4 (neglecting the I-N curve). This is illustrated by the simulation results. Judging from the snapshots in Fig. 2b and 2c it is clear that the columnar structures are robust against small orientational fluctuations. The differences between the layer pair correlation functions in Fig. 3 are very small. The number of lattice defects seems to increase slightly upon lowering the field strength but only at the smallest value $(\xi=10)$ do we observe that the hexagonal pattern has vanished completely. The system nevertheless displays significant liquid like order perpendicular to the director which is typical for a dense nematic state. In the absence of the field, the systems is completely isotropic, in qualitative accordance with the phase diagram in Fig. 4.

So far, we have implicitly assumed that the bifurcations represent thermodynamically stable phase transitions. Although the simulations clearly point to a (mechanically) stable columnar phase for both parallel and asymptotically rotating rods, it would be desirable to get similar confirmation from the theory. To that end we have conducted a parametric expansion of the free energy around the bifurcation, elaborated in Appendix B.

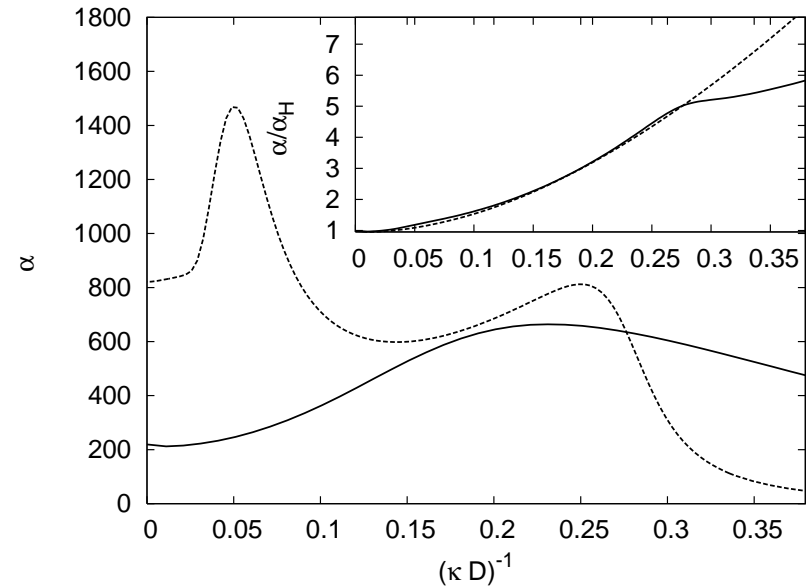

FIG. 5: Behavior of the Gaussian variational parameter $\alpha$ [see Eq. (40)] at the N-S (solid) and N-C (dotted) bifurcations as a function of Debye length. The inset shows the normalized $\tilde{\alpha}=\alpha / \alpha_{H}$ at the $\mathrm{N}-\mathrm{C}$ bifurcation.

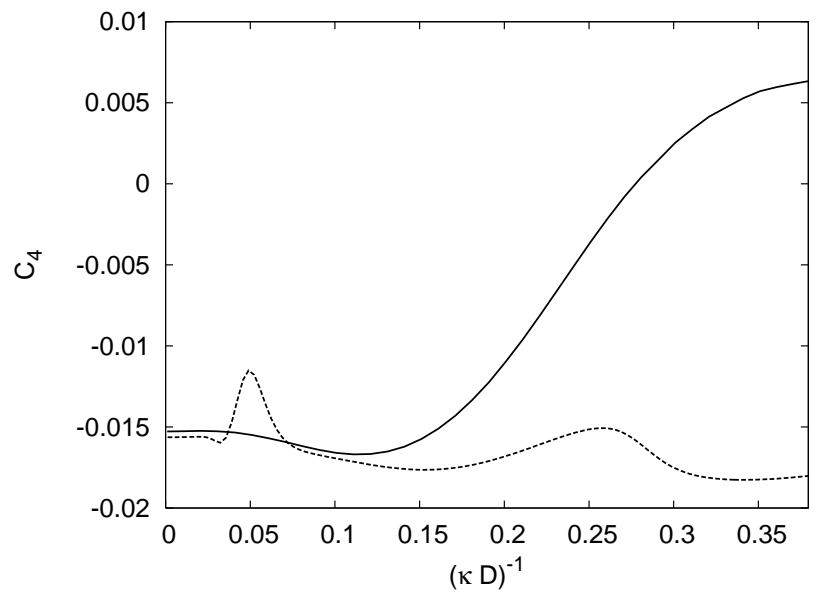

FIG. 6: Landau coefficient $C_{4}$ given by Eq. 65) at the N-S (solid) and N-C (dotted) bifurcations as a function of Debye length corresponding to Fig. 4.

The resulting Landau coefficient is depicted in Fig. 6 . and it is clear that the thermodynamic stability condition $C_{4}<0$ is generally fulfilled (except in the smectic region where $\mathrm{N}-\mathrm{S}$ is pre-empted by $\mathrm{N}-\mathrm{C}$ ). A similar outcome is found for the parallel case, not shown here.

\section{CONCLUSIONS}

An extensive bifurcation analysis is presented on translational symmetry-breaking instabilities in nematic systems of charged rods within Onsager's second virial theory. Starting from an artificial system of parallel charged rods, modelled via a hard-core Yukawa site model, nematic-smectic and nematic-(hexagonal) columnar instabilities are scrutinized as a function of the Debye 
length. The treatment is then extended towards the more realistic situation of rods with rotational degrees of freedom by employing an asymptotic Gaussian analysis of the orientation-dependent quantities. In both cases, distinct preferential columnar order is observed for moderate Debye screening lengths. As a supplement, Brownian dynamics simulations for the point Yukawa site model were carried out and the stability of columnar structures at low screening conditions and low particle concentrations is qualitatively reproduced. No evidence for crystalline order was found. Based on this, no attempt has been made in theory to seek possible nematic-crystal instabilities, which could in principle be scrutinized by imposing coupled smectic and columnar density modulations. Moreover, these type of instabilities are more likely to occur at very low screening conditions where the effective rod aspect-ratio (which incorporates the extent of the double layers) is no longer large enough to guarantee a stable nematic phase. In this region one may expect an isotropic fluid of rods to directly freeze into a crystalline lattice without intervention of nematic order ${ }^{2.3}$. These type of bifurcations are not addressed in this paper because the numerical effort involved in quantifying the necessary electrostatic end cap contributions for freely rotating rods is beyond the scale of the present calculations.

Looking at the experimental results for charged rods we may put forward that a crossover from smectic order to a more intricate ordered state has been observed in concentrated systems of TMV rods upon decreasing ionic strength ${ }^{17}$. However, at present it is not fully clear whether these structures are really columnar or represent three-dimensional crystalline order. More detailed structural investigations are probably required to resolve this ambiguity.

Our calculations also show that the columnar order can be realized at fairly low concentrations if the rods are rendered in near-parallel configurations by an external field. This could be any field that couples primarily to the rod orientations, for example magnetic, electric or shear flow fields. Finally, we remark that our results are connected to observations in other systems of rod-like mesogens with soft interactions. Columnar phases occur in complex systems of stiff polyelectrolytes like DNA $\underline{46,47}$ and may be induced by an external magnetic field in systems of dipolar colloids $\underline{48}$ and lath-shaped, goethite colloids $\underline{49}$. Although other complicating features such as length polydispersity, dipole-dipole or non-uniform sitesite interactions are at play in these systems, it is intriguing to see that similar columnar structures may occur in a relatively simple model system for soft rods considered here. Future work could be aimed at finding out whether a scenario such as in Fig. 1 is qualitatively reproducible for other soft potentials. It is also desirable to enlarge the scope of the simulations such that a wide area of concentrations and rod potentials can be covered and better comparisons with theory and experiments can be made.

\section{Appendix A: Isotropic-nematic bifurcation}

The bifurcation from the isotropic to the nematic state can be analytically derived starting from the stationarity condition Eq. (4) which we may rewrite as

$$
\ln f(\Omega)=\mu+\rho \int f\left(\Omega^{\prime}\right) \hat{\Phi}\left(0 ; \Omega, \Omega^{\prime}\right) d \Omega^{\prime}
$$

where the constant $\mu$ arises from the normalization condition of the ODF. The Mayer kernel at zero wavevector is related to the second virial coefficient of two charged rods [cf. Eq. (38)]:

$$
\hat{\Phi}\left(0 ; \Omega, \Omega^{\prime}\right)=-2 L^{2} D_{\text {eff }}\left(\Omega, \Omega^{\prime}\right)\left|\sin \gamma\left(\Omega, \Omega^{\prime}\right)\right|
$$

The contributions of $\mathcal{O}\left(L D^{2}\right)$ do not depend on orientation and hence drop out of the free energy minimization with respect to the ODF leading to Eq. (52). Close to the bifurcation point the ODF can be parametrized as:

$$
f(\Omega)=\frac{1}{4 \pi}\left[1+\epsilon \mathcal{P}_{2}(\cos \theta)\right]
$$

with $\mathcal{P}_{n}$ a Legendre polynomial and $\epsilon$ an arbitrarily small order parameter quantifying a uniaxial nematic perturbation to the isotropic ODF $f=1 / 4 \pi$. Likewise, the orientation-dependent functions in the Mayer kernel can be expanded in terms of these polynomials in the following way 21 :

$$
F(\sin \gamma)=c_{0}+c_{2} \mathcal{P}_{2}(\cos \theta) \mathcal{P}_{2}\left(\cos \theta^{\prime}\right)+\cdots
$$

with coefficients $c_{0}=\pi / 4$ and $c_{2}=-5 \pi / 32$ for $F(x)=x$ and $c_{0}=(\pi / 4)[\ln 2-1 / 2]$ and $c_{2}=(-5 \pi / 32)[\ln 2-5 / 4]$ for $F(x)=-x \ln x$. Inserting these into the stationarity condition Eq. (52), linearizing with respect to $\epsilon$ and some rearranging (using the orthogonality condition $\left.\int_{-1}^{1} d(\cos \theta) \mathcal{P}_{n}(\cos \theta) \mathcal{P}_{m}(\cos \theta)=2 \delta_{n m} /(2 n+1)\right)$ readily leads to a closed expression for the bifurcation concentration:

$$
c^{*}=\eta^{*} \frac{L}{D}=\frac{4}{1+(\kappa D)^{-1}\left(\ln A+\gamma_{E}-\ln 2-\frac{5}{4}\right)}
$$

with $A$ given by Eq. (28).

\section{Appendix B: Landau expansion around the bifurcation point}

An expression for the free energy difference $\Delta F=$ $F_{I}-F_{N}$ between the inhomogeneous state and the homogeneous nematic reference state can be obtained by inserting the parametrization Eq. (6) into the functional Eq. (11). After some rearranging one can show that the free energy difference per particle reads:

$$
\frac{\Delta \beta F}{N}=\frac{1}{2 \pi} \int_{0}^{2 \pi} d \zeta W(\zeta) \ln W(\zeta)-\frac{\rho}{4} \sum_{l=1}^{\infty} a_{l}^{2} \hat{\Phi}_{f}(l q)
$$


using the short-hand notation $\hat{\Phi}_{f}(l q)=\left\langle\left\langle\hat{\Phi}\left(l \mathbf{q} ; \Omega, \Omega^{\prime}\right)\right\rangle\right\rangle_{f}$ and

$$
W(\zeta)=1+\sum_{l=1}^{\infty} a_{l} \cos (l \zeta)
$$

For the sake of brevity we will restrict ourselves to the smectic case here, i.e. a single instability mode. The analysis for the columnar symmetry can be carried out in a similar manner. Minimizing with respect to the order parameters $a_{l}$ and the wave number $q$ leads to the following stability conditions

$$
\begin{aligned}
A\left(a_{l}, q, \rho\right)= & \frac{1}{2 \pi} \int_{0}^{2 \pi} d \zeta \cos (m \zeta) \ln W(\zeta) \\
& -\frac{\rho}{2} a_{m} \hat{\Phi}_{f}(m q)=0, \quad m \geq 1 \\
B\left(a_{l}, q, \rho\right)= & \rho \sum_{l=1}^{\infty} a_{l}^{2} \hat{\Phi}_{f}^{\prime}(l q)=0
\end{aligned}
$$

with $\hat{\Phi}^{\prime}=\partial \hat{\Phi} / \partial q$. Let us now propose the following expansions in terms of an arbitrarily small parameter $\epsilon$

$$
\begin{aligned}
W(\zeta ; \epsilon) & =1+\epsilon a_{1} \cos \zeta+\epsilon^{2} a_{2} \cos 2 \zeta+\epsilon^{3} a_{3} \cos 3 \zeta+\cdots \\
\rho(\epsilon) & =\rho_{0}+\epsilon \rho_{1}+\epsilon^{2} \rho_{2}+\cdots \\
q(\epsilon) & =q_{0}+\epsilon q_{1}+\epsilon^{2} q_{2}+\cdots
\end{aligned}
$$

The expansion of $W$ is justified close to bifurcation point where the spatial inhomogeneity is supposed to be weak. The zeroth order solution $\epsilon=0$ reproduces the bifurcation condition $\rho_{0} \hat{\Phi}_{f}(q)=1$ and hence $\left\{a_{1}, \rho_{0}, q_{0}\right\}=$ $\left\{1, \rho^{*}, q^{*}\right\}$. Inserting the parametrization into Eq. (59) allows us to expand the set of stationarity conditions as follows:

$$
\begin{aligned}
& A(\epsilon)=\epsilon\left(A_{m}^{(0)}+\epsilon A_{m}^{(1)}+\epsilon^{2} A_{m}^{(2)}+\cdots\right)=0, \quad m \geq 1 \\
& B(\epsilon)=\epsilon^{2}\left(B^{(0)}+\epsilon B^{(1)}+\epsilon^{2} B^{(2)}+\cdots\right)=0
\end{aligned}
$$

The calculation of the coefficients is straightforward but tedious and we will only give the essential results in the remainder of the Appendix. Performing an order by order solution of the above set of equations yields up to first order

$$
\rho_{1}=0
$$

$$
\begin{aligned}
q_{1} & =0 \\
a_{2} & =\frac{1}{4\left(1-\rho_{0} \hat{\Phi}_{f}(2 q)\right)}
\end{aligned}
$$

and up to second order (dropping the caret and the subscript $f$ for notational clarity)

$$
\begin{aligned}
\rho_{2} & =\frac{\frac{1}{8} \Phi^{\prime \prime}(q)\left(1-2 a_{2}\right)+\rho_{0} a_{2}^{2} \Phi^{\prime}(q) \Phi^{\prime}(2 q)}{\frac{1}{2} \Phi(q) \Phi^{\prime \prime}(q)-\left(\Phi^{\prime}(q)\right)^{2}} \\
q_{2} & =\frac{\frac{1}{4} \Phi^{\prime}(q)\left(1-2 a_{2}\right)+a_{2}^{2} \Phi^{\prime}(2 q)}{\rho_{0}(\Phi(q))^{2}-\frac{1}{2} \Phi^{\prime \prime}(q)} \\
a_{3} & =\frac{\frac{1}{2} a_{2}-\frac{1}{12}}{\rho_{0} \Phi(3 q)-1}
\end{aligned}
$$

The free energy difference can now be expanded in an analogous way using the parametrization Eq. (60) in Eq. (57). The Landau free energy as a function of the order parameter $\epsilon$ reads

$$
\frac{\Delta \beta F}{N}=\epsilon^{2} C_{2}+\epsilon^{3} C_{3}+\epsilon^{4} C_{4}
$$

with coefficients

$$
\begin{aligned}
C_{2} & =0 \\
C_{3} & =0 \\
C_{4} & =\frac{1}{64}\left(\frac{\rho_{0} \Phi(2 q)}{1-\rho_{0} \Phi(2 q)}-1\right)
\end{aligned}
$$

In agreement with Mulder's results 26 we get a zero cubic contribution in Eq. (64) indicating the transition towards the inhomogeneous state to be of second order. The thermodynamic stability of the new state however is guaranteed by the condition $C_{4}<0$ which has to be assessed numerically via the Mayer kernel.

\section{Acknowledgments}

I am grateful to R. Blaak for useful discussions and a critical reading of the manuscript. This work was supported by the Deutsche Forschungsgemeinschaft under SFB-TR6 and the Alexander von Humboldt Foundation.
* Electronic address: wensink@thphy.uni-duesseldorf.de

1 L. Onsager, Ann. N.Y. Acad. Sci. 51, 627 (1949).

2 S. C. McGrother, D. C. Williamson, and G. Jackson, J. Chem. Phys. 104, 6755 (1995).

3 P. Bolhuis and D. Frenkel, J. Chem. Phys. 106, 666 (1997).

${ }^{4}$ D. Frenkel, Liq. Cryst. 5, 929 (1989).

5 J. A. C. Veerman and D. Frenkel, Phys. Rev. A 45, 5632 (1992).
${ }^{6}$ A. B. D. Brown, C. Ferrero, T. Narayanan, and A. R. Rennie, Eur. Phys. J. B 11, 481 (1999).

7 F. M. van der Kooij, K. Kassapidou, and H. N. W. Lekkerkerker, Nature 406, 868 (2000).

8 A. R. Khokhlov and A. N. Semenov, Physica A 108, 546 (1981).

9 T. Odijk, Macromolecules 19, 2313 (1986).

10 G. J. Vroege and H. N. W. Lekkerkerker, Rep. Prog. Phys. 
55, $1241(1992)$

11 P. van der Schoot, J. Chem. Phys. 104, 1130 (1996).

12 M. P. Taylor and J. Herzfeld, Langmuir 6, 911 (1990).

13 A. Stroobants, Phys. Rev. Lett. 69, 14 (1992).

14 S.-M. Cui and Z. Y. Chen, Phys. Rev. E 50, 3747 (1994).

15 M. P. B. van Bruggen, M. Donker, H. N. W. Lekkerkerker, and T. L. Hughes, Colloids Surf., A 150, 115 (1999).

16 M. P. B. van Bruggen, Langmuir 14, 2245 (1998).

17 S. Fraden, in Observation, prediction and simulation of phase transitions in complex fluids, edited by M. Baus (Kluwer, 1995).

18 P. Davidon and J. C. P. Gabriel, Curr. Opin. Colloid Interface Sci. 9, 377 (2005).

19 B. J. Lemaire, P. Davidson, J. Ferré, J. P. Jamet, D. Petermann, P. Panine, I. Dozov, and J. P. Jolivet, Eur. Phys. J. E 13, 291 (2004).

${ }^{20}$ H. Zocher, Z. Anorg. Allg. Chem. 147, 91 (1925).

21 A. Stroobants, H. N. W. Lekkerkerker, and T. Odijk, Macromolecules 19, 2232 (1986).

22 E. M. Kramer and J. Herzfeld, Phys. Rev. E 61, 6872 (2000).

${ }^{23}$ H. Graf and H. Löwen, Phys. Rev. E 59, 1932 (1999).

24 E. M. Kramer and J. Herzfeld, J. Chem. Phys. 110, 8825 (1999).

25 B. M. Mulder, Phys. Rev. A 39, 360 (1989).

26 B. M. Mulder, Phys. Rev. A 35, 3095 (1987).

27 D. Frenkel, H. N. W. Lekkerkerker, and A. Stroobants, Nature 332, 822 (1988).

28 A. Poniewierski and R. Holyst, Phys. Rev. Lett. 61, 2461 (1988).

29 H. H. Wensink, Ph.D. thesis, Utrecht University (2004).

${ }^{30}$ R. F. Kayser and H. J. Raveche, Phys. Rev. A 17, 2067
(1978).

31 R. van Roij, P. Bolhuis, B. Mulder, and D. Frenkel, Phys. Rev. E 52 (1995).

32 T. Odijk, J. Chem. Phys. 93, 5172 (1990).

33 T. Kirchhoff, H. Löwen, and R. Klein, Phys. Rev. E 53, 5011 (1996).

${ }^{34}$ R. van Roij, Ph.D. thesis, Utrecht University (1996).

35 T. Odijk and H. N. W. Lekkerkerker, J. Phys. Chem. 89, 2090 (1985).

36 W. H. Press, et al., Numerical Recipes (Cambridge University Press, New York, 1989).

37 J. D. Parsons, Phys. Rev. A 19, 1225 (1979).

38 S. D. Lee, J. Chem. Phys. 87, 4972 (1987).

39 S. D. Lee, J. Chem. Phys. 89, 7036 (1989).

40 D. Frenkel, J. Phys. Chem. 92, 3280 (1988).

41 D. L. Ermak, J. Chem. Phys. 62, 4189 (1975).

42 M. M. Tirado, J. G. de la Torre, and C. L. Martinez, J. Chem. Phys. 81, 2047 (1984).

43 G. Cinacchi, L. D. Gaetani, and A. Tani, Phys. Rev. E 71, 031703 (2005).

44 M. Allen and D. Tildesley, Computer simulation of liquids (Clarendon, Oxford, 1987).

45 J. R. Philip and R. A. Wooding, J. Chem. Phys. 52, 953 (1970).

${ }^{46}$ F. Livolant, A. M. Levelut, J. Doucet, and J. P. Benoit, Nature 339, 724 (1989).

47 F. Livolant and A. Leforestier, Prog. Polym. Sci. 21, 1115 (1996).

48 C. Holm and J. J. Weis, Curr. Opin. Colloid Interface Sci. 10, 133 (2005).

49 B. J. Lemaire, P. Davidson, P. Panine, and J. P. Jolivet, Phys. Rev. Lett. 93, 267801 (2004). 